\title{
Evaluating Gaussian and Rayleigh-Based Mathematical Models for T and P-waves in ECG
}

\author{
J. P. V. Madeiro, E. M. B. Santos, P. C. Cortez, J. H. S. Felix, F. S. Schlindwein
}

\begin{abstract}
This paper presents a comparative study of modelling and segmentation of $P$ and $T$ waves in electrocardiograms, using three different mathematical models: Gaussian function, a composition of two Gaussian functions and Rayleigh probability density function (Rayleigh pdf). In order to evaluate the adaptability and the matching degree between each model and each characteristic wave, we compute the normalized root mean square (RMS) error, as well as the evolution of the fitting parameters related to each kernel throughout ECG records from the well-known QT database. Concerning the delineation of $P$ and $T$-waves, we apply Wavelet Transform for estimating $T$-wave and $P$-wave peak locations and combine each developed model with an approach based on the computation of Trapezium's area to locate the end point of each $T$-wave and the beginning and end point of each $P$-wave. The composition of two Gaussian functions has shown the most accurate results concerning wave modelling, providing average values of normalized RMS equal to $9,15 \%$ and $18,70 \%$, respectively for $T$ wave and $P$-wave, and the Rayleigh pdf provided the most stable fitting parameters. For $T$-wave end location, the most accurate results were computed when using the kernel composition of two Gaussian functions, for which the average time error was 4,49 \pm 14,32 ms. For $P$-wave begin and $P$-wave end locations, the most accurate results were computed when using kernel Rayleigh pdf, for which the average time errors were, respectively, $-4,23 \pm$ $14,84 \mathrm{~ms}$ and $2,26 \pm 13,14 \mathrm{~ms}$.
\end{abstract}

Keywords - electrocardiogram (ECG), $P$ and $T$ waves, mathematical models, normalized RMS error, Gaussian function, Rayleigh pdf.

\section{INTRODUÇÃO}

A partir do processamento automático do sinal eletrocardiograma (ECG) através de algoritmos específicos, extraem-se de cada ciclo cardíaco métricas que são utilizadas para o laudo descritivo do ECG, quais sejam: duração e amplitude da onda P, duração do intervalo PR (que vai do início da $\mathrm{P}$ até o início do complexo QRS), duração e amplitude do complexo QRS, alterações dos intervalos ST-T (que vai do final do complexo QRS ao final da onda T) e QT (que vai do início do complexo QRS ao final da onda T). A Figura 1 ilustra as referidas métricas, que são extraídas com o

J. P. V. Madeiro, Universidade da Integração Internacional da Lusofonia Afro-Brasileira (UNILAB), Redenção, Ceará, Brasil, jpaulo.vale@unilab.edu.br

E. M. B. Santos, Universidade da Integração Internacional da Lusofonia AfroBrasileira (UNILAB), Redenção, Ceará, Brasil, elvesfixe14@gmail.com

P. C. Cortez, Universidade Federal do Ceará (UFC), Fortaleza, Ceará, Brasil, cortez@lesc.ufc.br

J. H. S. Felix, Universidade da Integração Internacional da Lusofonia AfroBrasileira (UNILAB), Redenção, Ceará, Brasil, johnfelix@unilab.edu.br

F. S. Schlindwein, University of Leicester, Leicester, Leicestershire, Reino Unido, fss1@le.ac.uk. uso de algoritmos computacionais.

Além das tradicionais métricas temporais, a caracterização da morfologia das ondas características do ECG também proporciona a extração de informações significativas acerca da atividade cardíaca e pode sinalizar eventos associados a anomalias cardíacas que exijam intervenção médica emergencial [1,2]. Com relação à onda $T$, podem ser distinguidas seis possíveis morfologias: positiva $(+)$, negativa ou invertida (-), bifásica (+/- ou -/+), oscilação ascendente e oscilação descendente. Com relação à onda $\mathrm{P}$, quatro possíveis morfologias são admitidas: positiva, negativa e bifásicas (+/ou $-/+)[3,4]$.

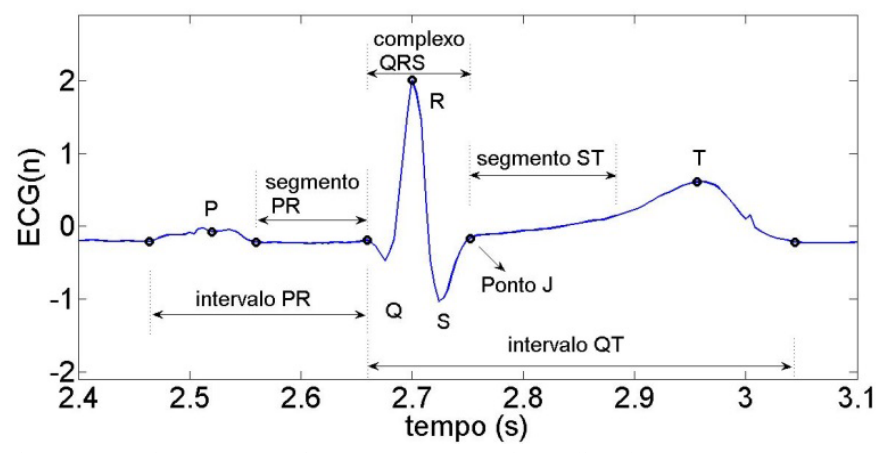

Figura 1. Ondas características e parâmetros de análise do ECG.

A onda T é normalmente assimétrica, de início mais lento e final mais rápido, positiva em quase todas as derivações, habitualmente com polaridade semelhante à do QRS e de amplitude equivalente a cerca de $10 \%$ a $30 \%$ do QRS [5]. A identificação de anormalidades incluem ondas $\mathrm{T}$ hiperagudas, invertidas, bifásicas, com forma de corcova de camelo (camelhump) e com achatamento. Tais alterações podem estar relacionadas, uma a uma, a diversos eventos importantes como: hipercalemia (sinalizado por ondas $\mathrm{T}$ positivas, simétricas e pontiagudas), isquemia subepicárdica (presença de onda $\mathrm{T}$ negativa, simétrica e pontiaguda), isquemia subendocárdica (presença de onda $\mathrm{T}$ positiva, simétrica e pontiaguda), alterações de repolarização ventricular (achatamento da onda $\mathrm{T}$ nas derivações esquerdas), infarto (elevação do segmento $\mathrm{ST}$ ), bloqueio de ramo (ondas $\mathrm{T}$ invertidas), embolia pulmonar (negativação de onda $\mathrm{T}$ ), cardiomiopatia arritmogênica do ventrículo direito (ondas $\mathrm{T}$ negativas), hipopotassemia (sinalizado por onda $\mathrm{T}$ bifásica) e acidente vascular cerebral isquêmico (ondas $\mathrm{T}$ invertidas com base larga) [6].

Já a onda $\mathrm{P}$ normal possui amplitude máxima de 2,5 mm (em que $1 \mathrm{~mm}$ equivale a $0,1 \mathrm{mV}$ ) e duração inferior a 110 ms, podendo ocorrer modificações de sua morfologia dependentes da frequência cardíaca. O ritmo sinusal do 


\section{THIS MODEL IS JUST A REFERENCE, THIS IS A PAPER FOR LATIN AMERICA IEEE \\ TRANSACTIONS.}

coração (fisiológico) é observado no ECG de superfície pela presença de ondas $\mathrm{P}$ positivas nas derivações I, II e aVF [5]. O impulso cardíaco normal inicia-se no nó sinusal e, então, espalha-se pela câmara atrial, definindo a forma da onda P. Contudo, quando a frente de onda de despolarização atrial se espalha de forma anormal e/ou apresenta caminhos acessórios, reentradas ou atrasos de condução, a morfologia da onda P é modificada [7]. Ondas P de duração mais longa e mais fragmentadas têm sido associadas a alterações da condução atrial, blocos de impulsos sinusais, aumento do átrio esquerdo e hipertensão [8]. Neste contexto, o prolongamento do tempo de condução e a propagação não homogênea dos impulsos sinusais são características eletrofisiológicas bem conhecidas do átrio propenso à fibrilação [9]. Portanto, a análise morfológica da onda $\mathrm{P}$ torna-se cada vez mais necessária para estratificação de risco acerca da predisposição de pacientes ao acometimento de fibrilação atrial, os quais poderiam se beneficiar de tratamentos eficazes preventivos [8].

A ideia de se utilizar funções matemáticas para modelar o comportamento das ondas características do sinal ECG já vem de longo tempo na literatura. Inicialmente, a utilização destas ferramentas visava fornecer subsídios para a análise espectral do sinal real, a partir de uma aproximação. Assim, propunhase um determinado modelo com um conjunto de parâmetros ajustáveis para a representação de cada ciclo cardíaco ou batimento, satisfazendo alguns pré-requisitos de precisão. $\mathrm{Na}$ abordagem desenvolvida por Richardson et al. [10], três modelos matemáticos são propostos para representar individualmente a onda P, o complexo QRS e a onda T, usando-se funções Gaussianas e suas primeira e segunda derivadas. Em técnicas de extração de parâmetros mais recentes, a modelagem matemática faz parte de uma estrutura de duplo estágio. Num primeiro estágio, derivam-se os parâmetros de uma função matemática que aproxima um determinado segmento do ciclo cardíaco, e num estágio subsequente realiza-se a caracterização morfológica das correspondentes formas de onda. Referida caracterização é desenvolvida através do uso de limiares, adaptativos ou não, aplicados sobre o modelo obtido [11-12].

O objetivo deste trabalho é propor e comparar diferentes kernels para modelagem e segmentação das ondas $\mathrm{P}$ e T com base em adaptações da função Gaussiana e da função densidade de probabilidade de Rayleigh. O desempenho de cada modelo matemático é avaliado com o processamento de todos os sinais publicamente disponíveis na base de dados QT/physionet [13], através do cálculo do erro RMS normalizado entre cada modelo e cada forma de onda analisada, das diferenças temporais entre as marcações automáticas de segmentação e marcações manuais, e da evolução dos parâmetros de configuração relacionados aos kernels modeladores ao longo dos sinais da base.

O presente artigo está estruturado como segue. Na seção II, são detalhados os modelos matemáticos propostos e a metodologia de deteç̧ão, modelagem e segmentação das ondas fisiológicas $\mathrm{P}$ e $\mathrm{T}$. Na seção III, ilustram-se os resultados obtidos de comparação de desempenho dos kernels tanto no que se refere à adaptação à gama diversificada de morfologias da base de dados QT, quanto à precisão e à acurácia no delineamento das ondas características. Na seção IV, é realizada a discussão dos resultados, e, finalmente, na seção V, são estabelecidas as conclusões derivadas do estudo realizado e as propostas de trabalhos futuros.

\section{MATERIAIS E MÉTODOS}

Este trabalho considera apenas a detecção, segmentação e modelagem das ondas P e T. Certamente, o complexo QRS precisa ser previamente detectado e segmentado, de forma a delimitar-se um intervalo de busca que contenha as ondas $\mathrm{P}$ e T. Foi adotada abordagem de detecção e segmentação de QRS previamente validada pelos autores, a qual é baseada nas transformadas de Hilbert e Wavelet, na função primeiraderivada e na técnica do limiar adaptativo [14].

\section{A. Descrição dos Modelos Matemáticos}

Neste trabalho, aplicam-se três diferentes kernels matemáticos visando à modelagem das ondas P e T do ECG: a função Gaussiana, uma composição de duas funções Gaussianas e a função densidade de Probabilidade de Rayleigh.

Seja $G_{\sigma_{1}}(t)$ uma função Gaussiana de tempo contínuo de parâmetro $\sigma_{1}$ variável com amplitude máxima normalizada, dada por:

$G_{\sigma_{1}}(t)=\exp \left(-\frac{t^{2}}{2 \sigma_{1}^{2}}\right)$

Define-se uma função Gaussiana de tempo-discreto $G_{\sigma_{1}}[k]$, compreendendo o intervalo $-3 \leq k \leq 3$, em uma resolução de $N_{T}^{(1)}$ amostras, para modelagem de onda $\mathrm{T}$, ou $N_{P}^{(1)}$ amostras, para modelagem de onda $\mathrm{P}$, com um desvio-padrão $\sigma_{1}$ variável:

$G_{\sigma_{1}}[k]=\exp \left(-\frac{k^{2}}{2 \sigma_{1}^{2}}\right)$

De forma a derivar uma composição de duas funções Gaussianas de tempo discreto, denominada $G_{\sigma_{1}, \sigma_{2}}[k]$, parte-se de duas funções Gaussianas de tempo discreto $G_{\sigma_{1}}[k]$ e $G_{\sigma_{2}}[k]$, respectivamente com desvios-padrões variáveis $\sigma_{1}$ e $\sigma_{2}$, ambas definidas para $-3 \leq k \leq 3$, tal que:

$G_{\sigma_{1}, \sigma_{2}}[k]=\left\{\begin{array}{c}G_{\sigma_{1}}[k],-3 \leq k \leq 0 \\ G_{\sigma_{2}}[k], 0 \leq k \leq 3\end{array}\right.$.

Finalmente, define-se uma função densidade de probabilidade de Rayleigh de tempo discreto $R_{\sigma_{1}}[k]$, compreendendo o intervalo $0 \leq k \leq 10$, em uma resolução de $N_{T}^{(2)}$ amostras, para modelagem de onda $\mathrm{T}$, ou $N_{P}^{(2)}$ amostras, para modelagem de onda $\mathrm{P}$, com um desvio-padrão $\sigma_{1}$ variável: 


\section{THIS MODEL IS JUST A REFERENCE, THIS IS A PAPER FOR LATIN AMERICA IEEE}

TRANSACTIONS.

$R_{\sigma_{1}}[k]=\frac{k}{\sigma_{1}^{2}} \exp \left(-\frac{k^{2}}{2 \sigma_{1}^{2}}\right)$.

Adicionalmente, define-se uma modificação, $R_{\sigma_{1}}^{\prime}[k]$, da função densidade de probabilidade de Rayleigh, também compreendendo o intervalo $0 \leq k \leq 10$, dada como sua reflexão temporal, tal que:

$R_{\sigma_{1}}^{\prime}[k]=R_{\sigma_{1}}[10-k]$

Apresentam-se nas Figuras 2(a), 2(b), 2(c) e 2(d) os quatro modelos matemáticos propostos e detalhados para modelagem e segmentação das ondas $\mathrm{P}$ e T, considerando-se os valores de parâmetros: $\sigma_{1}=0,5$ e $N_{T}^{(1)}=80$ amostras, para a função $G_{\sigma_{1}}[k]$ (Figura 2(a)), $\sigma_{1}=0,7, \sigma_{2}=0,3$ e $N_{T}^{(1)}=80$ amostras para a função $G_{\sigma_{1}, \sigma_{2}}[k]$ (Figura 2(b)), $\sigma_{1}=0,5$, e $N_{T}^{(2)}=80$ para as funções $R_{\sigma_{1}}[k]$ e $R_{\sigma_{1}}^{\prime}[k]$ (Figuras 2(c) e 2(d)).

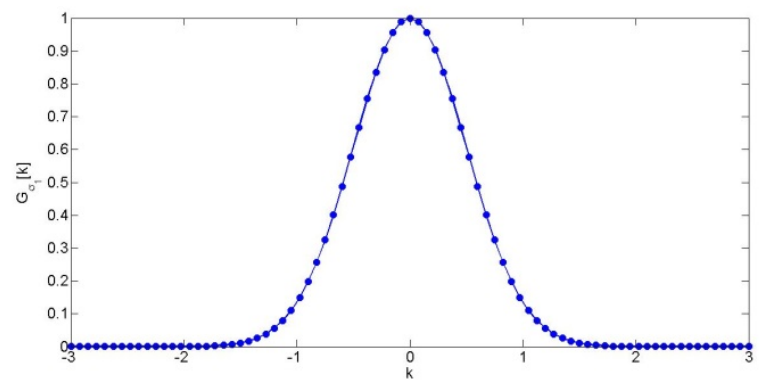

(a)

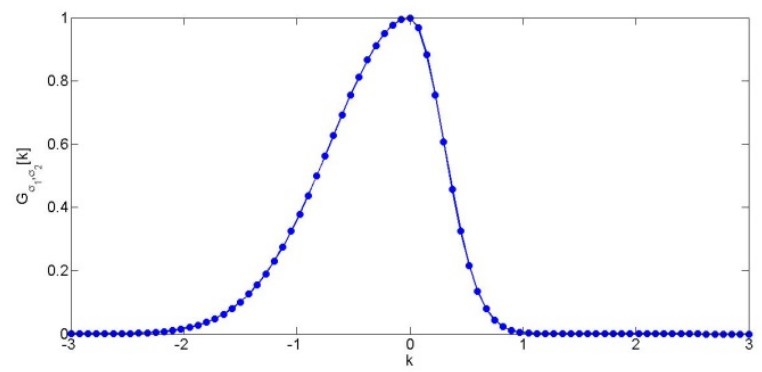

(b)

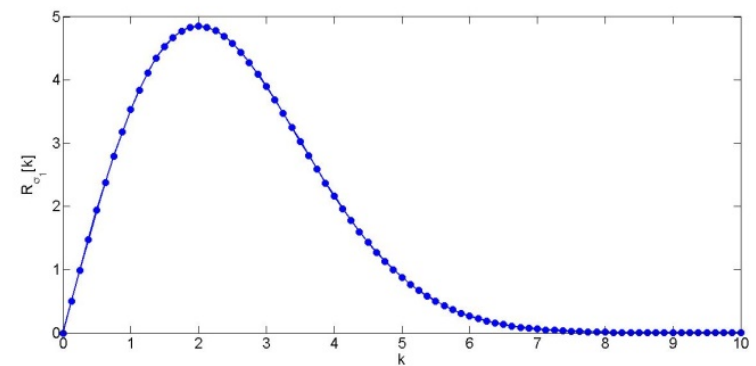

(c)

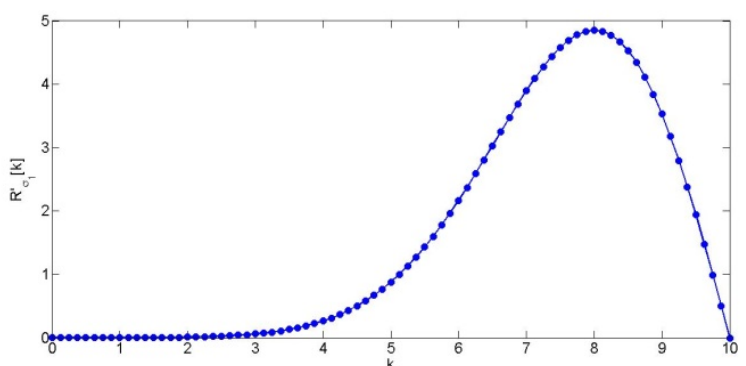

(d)

Figura 2. Detalhamento dos kernels matemáticos propostos para modelagem e segmentação das ondas P e T: (a) função Gaussiana de tempo discreto, $G_{\sigma_{1}}[k]$, $\sigma_{1}=0,5, N_{T}^{(1)}=80$, (b) composição de duas Gaussianas, $G_{\sigma_{1}, \sigma_{2}}[k], \sigma_{1}=0,7$ e $\sigma_{2}=0,3, N_{T}^{(1)}=80$, (c) função densidade de probabilidade de Rayleigh, $R_{\sigma_{1}}[k], \sigma_{1}=0,5, N_{T}^{(2)}=80$ e (e) versão refletida da função densidade de probabilidade de Rayleigh, $R_{\sigma_{1}}^{\prime}[k], \sigma_{1}=0,5, N_{T}^{(2)}=80$.

\section{B. Deteç̧ão do pico da onda T}

Para a detecção dos picos das ondas $\mathrm{P}$ e $\mathrm{T}$, aplica-se com algumas adaptações a abordagem desenvolvida por Martínez et al. [4], a qual considera, para sinais de tempo discreto, a transformada Wavelet discreta diádica (DWT), equivalente a um banco de filtros representado como uma cascata de filtros FIR passa-baixa e passa-alta, tal como ilustrado na Figura 1.

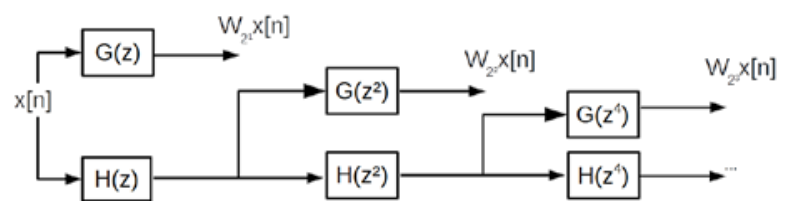

Figura 3: Implementação por banco de filtros da transformada DWT.

Como sugerido por Martínez et al. [4], usa-se como waveletmãe $\Psi(t)$ um spline quadrático, cuja transformada de Fourier é dada por

$\Psi(\Omega)=j \Omega\left(\frac{\sin \left(\frac{\Omega}{4}\right)}{\frac{\Omega}{4}}\right)^{4}$.

Esta função wavelet pode ser identificada como a derivada da convolução de quatro pulsos retangulares, i.e., a derivada de uma função passa-baixa.

As respostas ao impulso dos filtros $G(z)$ e $H(z)$ são dadas por [4]:

$g[n]=2 .\{\delta[n+1]-\delta[n]\}$,

$h[n]=\frac{1}{8} \cdot\{\delta[n+2]+3 \delta[n+1]+3 \delta[n]+\delta[n-1]\}$.

Primeiramente, define-se uma janela de busca para cada onda $\mathrm{T}$, relativamente a cada intervalo entre um dado ponto final de QRS e o subsequente ponto inicial de QRS. Sobre esta janela, busca-se por dois máximos locais de $\left|W_{2^{4}} x[n]\right|$. Se ao menos dois pontos críticos de sinais opostos excederem um limiar $\epsilon_{T}$, 


\section{THIS MODEL IS JUST A REFERENCE, THIS IS A PAPER FOR LATIN AMERICA IEEE TRANSACTIONS.}

calculado como 0,25. $R M S\left(W_{2^{4}} x[n]\right)$, o intervalo temporal entre eles não exceda $200 \mathrm{~ms}$ e a localização do primeiro deles não exceda $65 \%$ do intervalo temporal de busca, uma onda $\mathrm{T}$ é considerada presente. Caso acima de dois pares de pontos críticos satisfaçam tais condições, toma-se aquele cujo produto das duas amplitudes seja o maior dentre as calculadas. Neste caso, verifica-se se as amplitudes do par de pontos críticos detectados também excedem o limiar $\gamma_{T}$, calculado como $0,125 \cdot \max \left(\left|W_{2^{4}} x[n]\right|\right)$. Caso ambas as amplitudes excedam, os pontos críticos são mapeados como inclinações significativas no sinal original, e o cruzamento por zero localizado na região intermediária é mapeado como o pico da onda T. Caso apenas uma das amplitudes exceda o referido limiar, constata-se que a forma de onda detectada possui apenas uma inclinação significativa (morfologia ascendente/upward ou descendente/downward), e toma-se como referência de pico da onda o ponto de máximo ou ponto de mínimo de maior amplitude no sinal original localizado entre 0 instante do primeiro cruzamento por zero anteriormente à inclinação significativa em $W_{2^{4}} x[n]$ e o instante do primeiro cruzamento por zero posteriormente à inclinação significativa em $W_{2^{4}} x[n]$.

\section{Modelagem da onda T}

Após a determinação de cada pico de onda T, realiza-se o processo de modelagem da onda T. Cada um dos kernels propostos possui um ou dois parâmetros específicos, correspondendo aos desvios-padrões $\sigma_{1}$ e $\sigma_{2}$. Para cada kernel, estabelece-se um processo de adaptação ou conformação com cada forma de onda, variando-se os correspondentes parâmetros sobre uma faixa predefinida: $\sigma_{i} \in[0,2 ; 1,5]$, em que $i=1$, 2, e com resolução temporal de $N_{T}^{(1)}=N_{T}^{(1)}=80$ amostras.

Portanto, com relação ao kernel $G_{\sigma_{1}}[k]$, estabelece-se uma janela $W_{1}[n]$ em torno de cada pico de onda $\mathrm{T}$, iniciando 150 ms antes do correspondente ponto fiducial e finalizando 150 ms após o pico. Em seguida, aplica-se um processo de normalização das amostras do kernel, de forma que:

a) Sua amplitude máxima deve ser igual à máxima amplitude de $\left|W_{1}[n]\right| ;$

b) Sua mínima amplitude tomada anteriormente ao seu ponto de máximo deve ser igual ao primeiro ponto de mínimo de $\left|W_{1}[n]\right|$, i.e., a menor amplitude considerada do início ao ponto de máximo;

c) Sua mínima amplitude tomada posteriormente ao seu ponto de máximo deve ser igual ao segundo mínimo de $\left|W_{1}[n]\right|$, i.e., a menor amplitude considerada do ponto de máximo à última amostra.

Tal como detalhado, as amostras de cada kernel são normalizadas em dois estágios. Primeiramente, normalizam-se da amostra inicial à amostra correspondente ao ponto de máximo, de acordo com as amplitudes mínima e máxima de $\left|W_{1}[n]\right|$, consideradas também da amostra inicial ao ponto de máximo. Posteriormente, normalizam-se as amostras de um dado kernel analisado da posição correspondente ao ponto de máximo à última amostra, de acordo com as amplitudes máxima e mínima de $\left|W_{1}[n]\right|$ consideradas do ponto de máximo à amostra final.

Então, o kernel normalizado $G_{\sigma_{1}}^{m}[k]$ é alinhado pela localização da sua amplitude de pico com o pico de onda T na janela $W_{1}[n]$. Finalmente, como sugerido por Martínez et al. [2], calcula-se o erro RMS normalizado $E_{\sigma_{1}}^{(1)}$ para cada parâmetro $\sigma_{1}$ testado e cada forma de onda analisada, dado por

$E_{\sigma_{1}}^{(1)}=\sqrt{\frac{\sum_{k=P_{k}-\gamma}^{P_{k}^{+\gamma}}\left|W_{1}[k]-G_{\sigma_{1}}^{m}[k]\right|^{2}}{\sum_{k=P_{k}-\gamma}^{P_{k}+\gamma}\left|W_{1}[k]\right|^{2}}}$,

em que $P_{k}$ corresponde à posição do pico da onda $\mathrm{T}$ dentro da janela $W_{1}[n]$ e $\gamma$ refere-se ao número de amostras correspondentes a $150 \mathrm{~ms}$.

Portanto, realiza-se a pesquisa automática pelo valor de $\sigma_{1}$ relacionado ao valor mínimo de $E_{\sigma_{1}}^{(1)}$, denominado $E_{\text {min }}^{(1)}$, o qual refere-se ao erro RMS normalizado entre o kernel ótimo (melhor adaptado) e uma dada onda característica.

Analogamente, procede-se com as etapas de normalização de amplitude e alinhamento de pico para os demais kernels propostos, i.e., $G_{\sigma_{1}, \sigma_{2}}[k], R_{\sigma_{1}}[k], R_{\sigma_{1}}^{\prime}[k]$. O cálculo dos respectivos parâmetros de avaliação $E_{\sigma_{1}, \sigma_{2}}^{(2)}, E_{\sigma_{1}}^{(3)}$ e $E_{\sigma_{1}}^{(4)}$ e a busca pelos kernels ótimos são também processos análogos, atentando-se para o caso específico de $E_{\sigma_{1}, \sigma_{2}}^{(2)}$, em que todas as possíveis combinações de $\sigma_{1}$ e $\sigma_{2}$ devem ser avaliadas. Então, para cada sinal ECG analisado, calculam-se os valores médios dos parâmetros $E_{m i n}^{(1)}, E_{m i n}^{(2)}, E_{m i n}^{(3)}, E_{m i n}^{(4)}, E_{m i n}^{(5)}$, isto é, os correspondentes valores mínimos de erros RMS normalizados, bem como os respectivos desvios-padrões. Adicionalmente, são determinados para cada sinal ECG analisado a média e o desvio-padrão dos parâmetros ótimos $\sigma_{1}$ e/ou $\sigma_{2}$ relacionados a cada onda $\mathrm{T}$.

Como ilustrações da técnica proposta, as Figuras 4(a), 4(b), 4(c) e 4(d) apresentam processos de modelagem da onda $\mathrm{T}$ utilizando-se respectivamente os kernels $G_{\sigma_{1}}[k], G_{\sigma_{1}, \sigma_{2}}[k]$, $R_{\sigma_{1}}[k], R_{\sigma_{1}}^{\prime}[k]$, considerando o registro sel1457 da base QTDB.
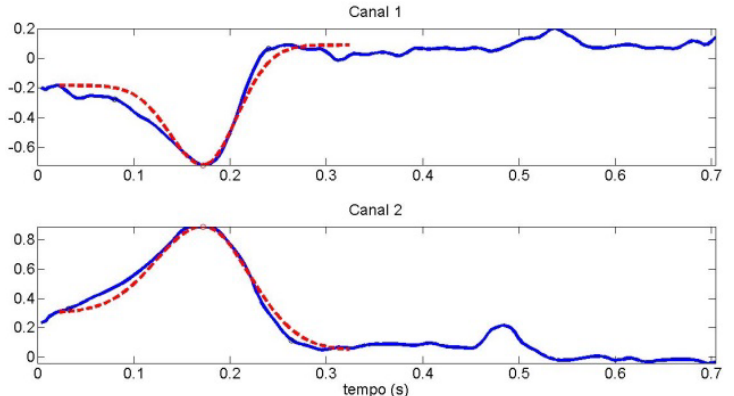

(a) 

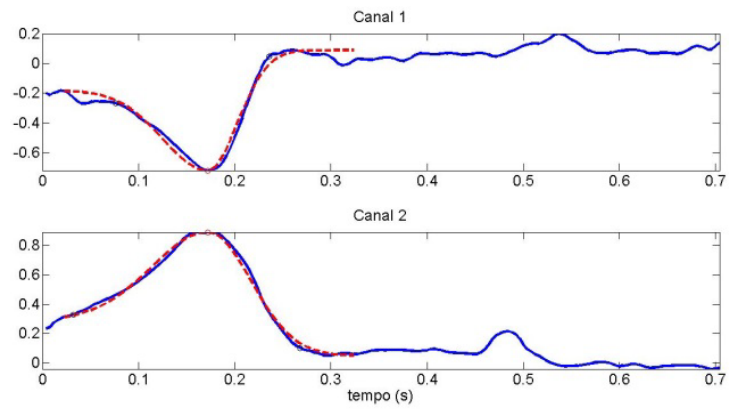

(b)
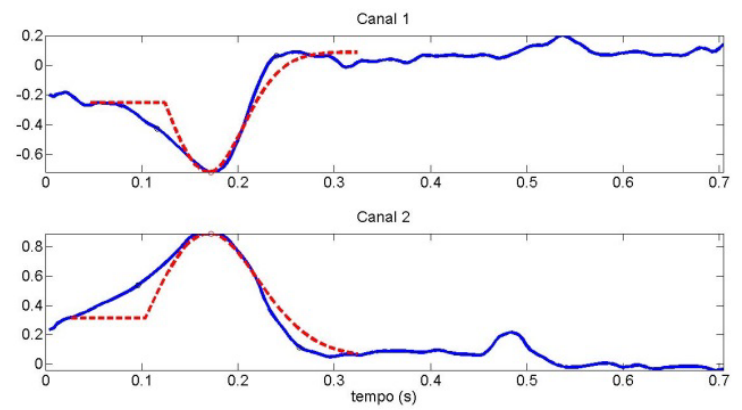

(c)
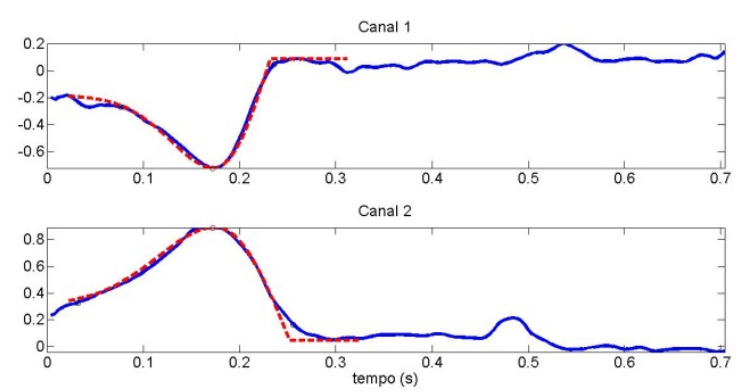

(d)

Figura 8 - Modelagem da onda T do registro sel14157 (canais 1 e 2) da base QTDB utilizando-se os kernels: (a) função gaussiana $G_{\sigma_{1}}[k]$, (b) composição de funções gaussianas $G_{\sigma_{1}, \sigma_{2}}[k]$, (c) função densidade de probabilidade de Rayleigh $R_{\sigma_{1}}[k]$, (d) versão refletida da função densidade de probabilidade de Rayleigh, kernel $R_{\sigma_{1}}^{\prime}[k]$.

\section{Delineamento da onda $T$}

O método de identificação das amostras relacionadas ao início e ao final da onda $\mathrm{T}$, conforme visto a seguir, apoia-se em pontos de amostra estratégicos de cada um dos modelos matemáticos, conforme sugerido por Madeiro et al. especificamente para a identificação do final da onda $\mathrm{T}$ [12].

Assim, executa-se a seguinte sequência de passos para a identificação do final da onda T:

(i) Busca-se num dado modelo matemático ótimo obtido, $G_{\sigma_{1}}[k], \quad G_{\sigma_{1}, \sigma_{2}}[k], \quad R_{\sigma_{1}}[k] \quad$ ou $\quad R_{\sigma_{1}}^{\prime}[k], \quad$ a amostra correspondente ao valor absoluto máximo da primeiraderivada, dentre aquelas amostras localizadas posteriormente à amplitude de pico do modelo matemático. Denomina-se a amostra assim identificada e o correspondente valor máximo da primeira derivada, respectivamente, como $T_{d e r}$ e $M_{d e r}$; (ii) Dentre as amostras localizadas após $T_{\text {der }}$, busca-se a primeira amostra cujo valor absoluto da primeira derivada esteja abaixo de 5\% do valor de $M_{d e r}$, sendo denominada a correspondente amostra assim localizada como $T_{\text {min }}$;

(iii) As amostras $T_{d e r}$ e $T_{\min }$ são mapeadas na janela de sinal $W_{1}[n]$ e, então, aplica-se a metodologia do cálculo da área de trapézios.

Define-se um índice de amostra móvel $T_{e}$, em que $T_{d e r} \leq$ $T_{e} \leq T_{\text {min }}$. Gera-se um trapézio definido pelos seguintes pares $(x, y): \quad\left(T_{d e r}, W_{1}\left[T_{d e r}\right]\right), \quad\left(T_{e}, W_{1}\left[T_{e}\right]\right),\left(T_{m i n}, W_{1}\left[T_{e}\right]\right) \quad$ e $\left(T_{\min }, W_{1}\left[T_{d e r}\right]\right)$. A área do trapézio, assim definido, é dada por [12]:

$$
A_{T}\left(T_{e}\right)=0,5\left|W\left[T_{d e r}\right]-W\left[T_{e}\right]\right|\left(2 T_{\min }-T_{e}-T_{d e r}\right) .
$$

A amostra correspondente ao final da onda $\mathrm{T}$ em análise é definida como aquela cujo valor de área $A_{T}\left(T_{e}\right)$ é máximo, denominando-se referida amostra como $T_{\text {end }}[6,7]$.

Para a localização do início da onda $\mathrm{T}$, executa-se uma sequência de passos análoga, tomando-se como referência as regiões do sinal de cada modelo matemático ótimo e da janela $W_{1}[n]$ localizadas anteriormente às suas respectivas amplitudes de pico:

(i) Busca-se num dado modelo matemático ótimo obtido, $G_{\sigma_{1}}[k], \quad G_{\sigma_{1}, \sigma_{2}}[k], \quad R_{\sigma_{1}}[k] \quad$ ou $R_{\sigma_{1}}^{\prime}[k], \quad$ a amostra correspondente ao valor absoluto máximo da primeiraderivada, dentre aquelas amostras localizadas anteriormente à amplitude de pico do modelo matemático. Denomina-se a amostra assim identificada e o correspondente valor máximo da primeira derivada, respectivamente, como $T_{d e r}$ e $M_{d e r}$;

(ii) Dentre as amostras localizadas antes de $T_{d e r}$, busca-se a última amostra cujo valor absoluto da primeira derivada esteja abaixo de $5 \%$ do valor de $M_{d e r}$, sendo denominada a correspondente amostra assim localizada como $T_{\text {min }}$;

(iii) As amostras $T_{\text {der }}$ e $T_{\text {min }}$ são mapeadas na janela de sinal $W_{1}[n]$ e, então, aplica-se a metodologia do cálculo da área de trapézios.

Define-se um índice de amostra móvel $T_{b}$, em que $T_{\min } \leq$ $T_{b} \leq T_{\text {der }}$. Gera-se um trapézio definido pelos seguintes pares $(x, y): \quad\left(T_{d e r}, W_{1}\left[T_{d e r}\right]\right), \quad\left(T_{b}, W_{1}\left[T_{b}\right]\right),\left(T_{\text {min }}, W_{1}\left[T_{b}\right]\right) \quad$ e $\left(T_{\text {min }}, W_{1}\left[T_{d e r}\right]\right)$. A área do trapézio, assim definido, é dada por:

$A_{T}\left(T_{b}\right)=0,5\left|W\left[T_{d e r}\right]-W\left[T_{b}\right]\right|\left(T_{d e r}+T_{b}-2 T_{\min }\right)$.

A amostra correspondente ao início da onda $\mathrm{T}$ em análise é definida como aquela cujo valor de área $A_{T}\left(T_{b}\right)$ é máximo, denominando-se referida amostra como $T_{\text {ini }}$.

Como ilustrações da técnica proposta, a Figura 9(a) apresenta o processo de segmentação da onda T para o registro sel14157 da base QTDB utilizando-se o kernel $G_{\sigma_{1}}[k]$. Analogamente, a Figura 5(b) apresenta o processo de segmentação da onda $\mathrm{T}$ para o mesmo registro utilizando-se o kernel $G_{\sigma_{1}, \sigma_{2}}[k]$, a Figura 5(c) apresenta o processo segmentação utilizando-se o 


\section{THIS MODEL IS JUST A REFERENCE, THIS IS A PAPER FOR LATIN AMERICA IEEE TRANSACTIONS.}

kernel $R_{\sigma_{1}}[k]$, e, finalmente, a Figura 5(d) apresenta os processo de segmentação utilizando-se o kernel $R_{\sigma_{1}}^{\prime}[k]$.
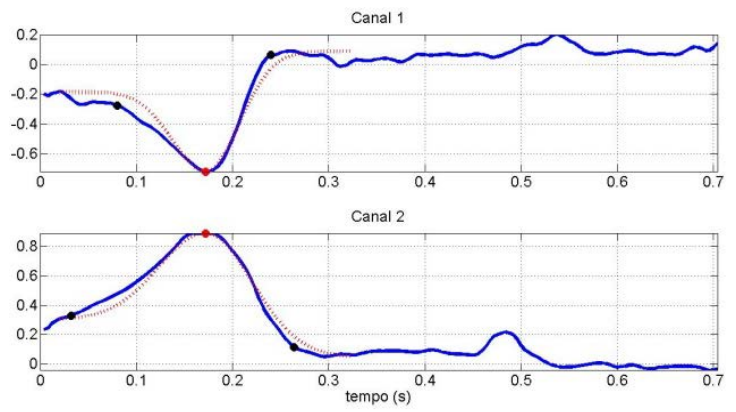

(a)
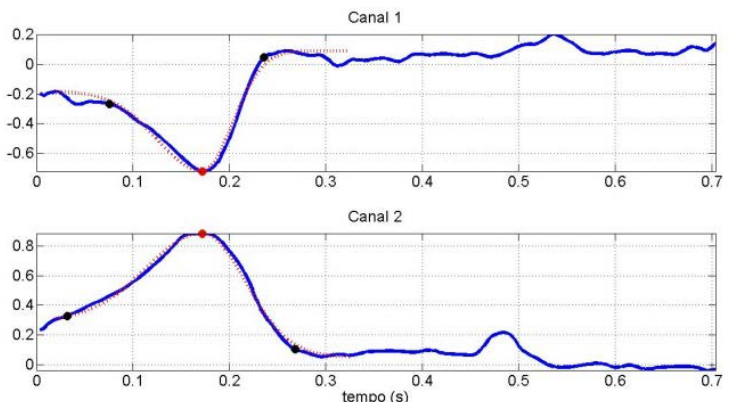

(b)

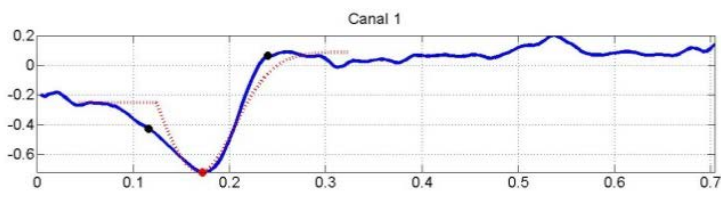

Canal 2

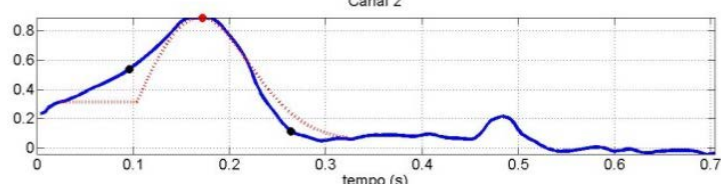

(c)

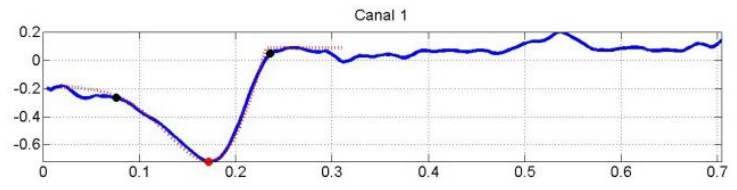

Canal 2

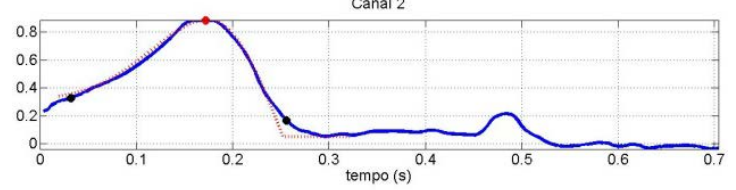

(d)

Figura 9 - Segmentação da onda T do registro sel14157 (canais 1 e 2) da base QTDB utilizando-se os kernels: (a) função gaussiana $G_{\sigma_{1}}[k]$, (b) composição de funções gaussianas $G_{\sigma_{1}, \sigma_{2}}[k]$, (c) função densidade de probabilidade de Rayleigh $R_{\sigma_{1}}[k]$, (d) versão refletida da função densidade de probabilidade de Rayleigh, kernel $R_{\sigma_{1}}^{\prime}[k]$.

\section{E. Identificação do pico da onda P}

Após os processos de detecção de pico, modelagem e delineamento de cada onda $T$, uma nova janela de busca é estabelecida para cada onda $\mathrm{P}$, relativamente a cada intervalo entre um dado ponto final de onda $\mathrm{T}$ e o subsequente ponto inicial de QRS. Analogamente como realizado para a busca de cada pico de onda $\mathrm{T}$, busca-se, sobre esta janela, por dois máximos locais de $\left|W_{2^{4}} x[n]\right|$. Se ao menos dois pontos críticos de sinais opostos excederem um limiar $\epsilon_{P}$, calculado como 0,02. $R M S\left(W_{2^{4}} x[n]\right)$, o intervalo temporal entre eles não exceda 200 ms e a localização do último deles não ocorra após $30 \mathrm{~ms}$ do final do intervalo de busca, uma onda P é considerada presente. Caso acima de dois pares de pontos críticos satisfaçam tais condições, toma-se aquele cujo produto das duas amplitudes seja o maior dentre as calculadas. Então, os pontos críticos selecionados são mapeados como inclinações significativas no sinal original, e o cruzamento por zero localizado na região intermediária é mapeado como o pico da onda P.

\section{F. Modelagem e delineamento da onda P}

Os processos de modelagem matemática e delineamento da onda $\mathrm{P}$ são análogos aos respectivos processos referentes à onda T. Estabelece-se uma janela $W_{2}[n]$ em torno de cada pico de onda $\mathrm{P}$, iniciando $100 \mathrm{~ms}$ antes do correspondente ponto fiducial e finalizando $100 \mathrm{~ms}$ após o pico. Para cada um dos kernels propostos, os quais possuem um ou dois parâmetros específicos, correspondendo aos desvios-padrões $\sigma_{1}$ e $\sigma_{2}$, estabelece-se um processo de adaptação ou conformação com cada forma de onda, variando-se os correspondentes parâmetros sobre uma faixa predefinida: $\sigma_{i} \in$ $[0,2 ; 1,5]$, em que $i=1$, 2 , e com resolução temporal de $N_{P}^{(1)}=$ $N_{P}^{(2)}=60$ amostras.

O processo de normalização das amostras de cada kernel, em dois estágios, dá-se de forma idêntica ao processo realizado para a onda $\mathrm{T}$, bem como o alinhamento de cada kernel normalizado pela localização de sua amplitude de pico com o pico de onda $\mathrm{P}$ na janela $W_{2}[n]$. O cálculo do erro RMS normalizado para cada parâmetro ou combinação de parâmetros de um dado kernel também é dado pela Equação 8, em que $P_{k}$ corresponde à posição do pico da onda $\mathrm{P}$ dentro da janela $W_{2}[n]$ e $\gamma$ refere-se ao número de amostras correspondentes a $100 \mathrm{~ms}$.

Finalmente, o método de identificação das amostras relacionadas ao início e ao final da onda $\mathrm{P}$, assim como para a onda $\mathrm{T}$, também apoia-se em pontos de amostra estratégicos de cada um dos modelos matemáticos. Assim, a sequência de passos utilizada para a identificação do início e final de cada onda $\mathrm{P}$, bem como as equações características de cálculo de área do trapézio (equações 9 e 10) são idênticas àquelas utilizadas respectivamente para identificação do inicío e final da onda $\mathrm{T}$.

Como ilustrações da técnica proposta, as Figuras 10(a) e 10(b) apresentam respectivamente os processos de modelagem e segmentação da onda P para o registro sel14157 da base QTDB utilizando-se o kernel $G_{\sigma_{1}}[k]$. Analogamente, as Figuras 10(c) e 10(d) apresentam os processos de modelagem 


\section{THIS MODEL IS JUST A REFERENCE, THIS IS A PAPER FOR LATIN AMERICA IEEE TRANSACTIONS.}

e segmentação da onda $\mathrm{P}$ para o mesmo registro utilizando-se o kernel $G_{\sigma_{1}, \sigma_{2}}[k]$, as Figuras 10(e) e 10(f) apresentam os processos de modelagem e segmentação utilizando-se o kernel $R_{\sigma_{1}}[k]$, e, finalmente, as figuras $10(\mathrm{~g})$ e $10(\mathrm{~h})$ apresentam os processos de modelagem e segmentação utilizando-se o kernel $R_{\sigma_{1}}^{\prime}[k]$.
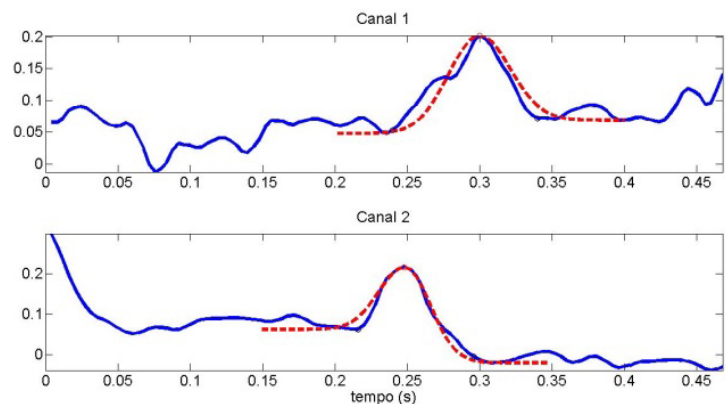

(a)
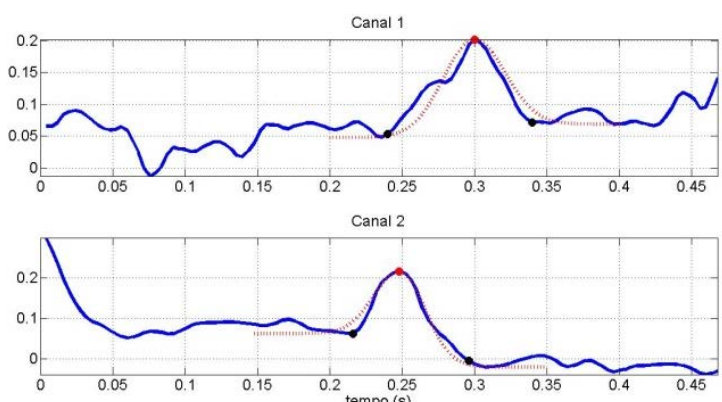

(b)
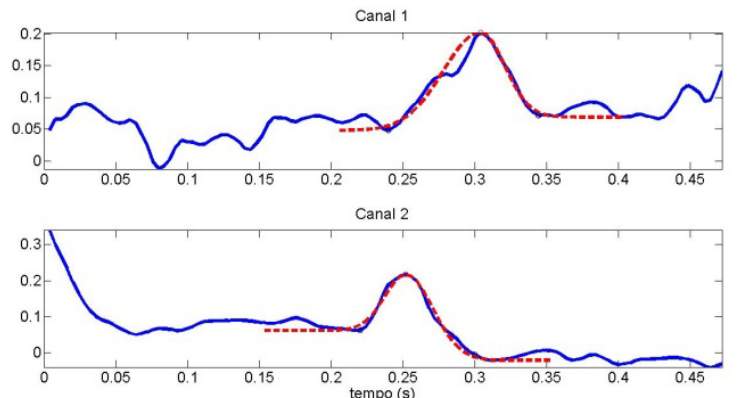

(c)
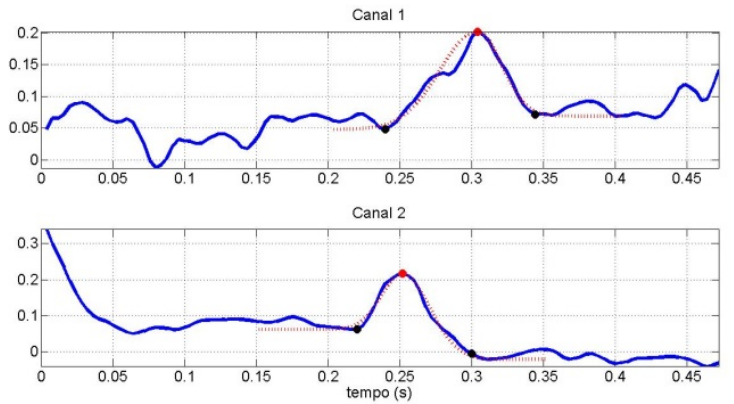

(d)
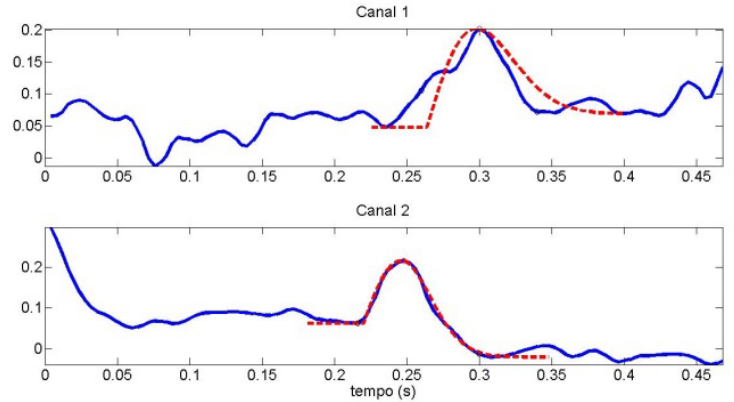

(e)
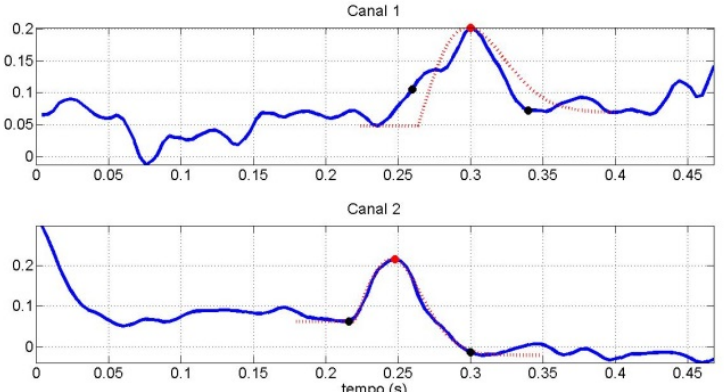

(f)
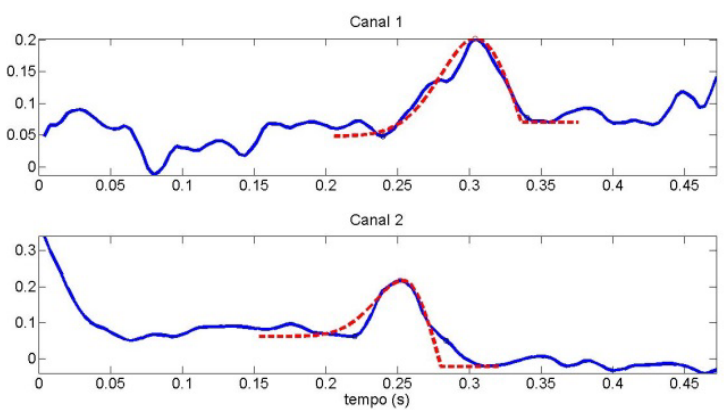

(g)
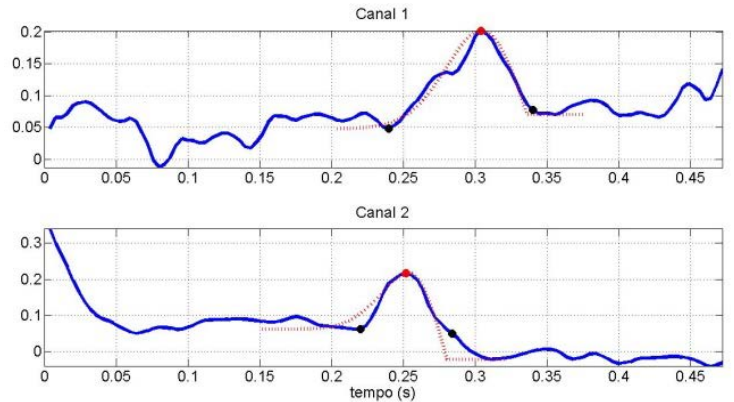

(h)

Figura 10 - Modelagem e segmentação da onda P do registro sel14157 (canais 1 e 2) da base QTDB utilizando-se os kernels: (a) e (b) função gaussiana $G_{\sigma_{1}}[k]$, (c) e (d) composição de funções gaussianas $G_{\sigma_{1}, \sigma_{2}}[k]$, (e) e (f) função densidade de probabilidade de Rayleigh $R_{\sigma_{1}}[k]$, (g) e (h) versão refletida da função densidade de probabilidade de Rayleigh, kernel $R_{\sigma_{1}}^{\prime}[k]$.

\section{RESULTADOS}

Os algoritmos propostos de modelagem e segmentação automática das ondas P e T do ECG são avaliados utilizandose a base de dados pública QT database (QTDB), a qual foi desenvolvida para validação de algoritmos de segmentação 


\section{THIS MODEL IS JUST A REFERENCE, THIS IS A PAPER FOR LATIN AMERICA IEEE TRANSACTIONS.}

das ondas características do ECG [13]. A base dispõe de 105 sinais ECG, cada um com duração de 15 minutos e frequência de amostragem de $250 \mathrm{~Hz}$. Inclui registros da base MIT-BIH Arrhythmia database e também de diversas outras bases do sistema Physionet (European ST-T database, ST change, Supraventricular Arrhythmia, Normal Sinus Rhythm, Sudden Death e Long Term) [13]. Anotações manuais de cardiologistas são disponibilizadas para, pelo menos, 30 batimentos por registro, incluindo-se os picos (pontos fiduciais) dos complexos QRS e das ondas P e T, inícios e finais das formas de onda.

Primeiramente, são comparados os valores médios e os desvios-padrões globais (considerando todos os sinais da base QTDB) dos erros RMS normalizados obtidos por cada um dos modelos ótimos correspondentes a cada kernel testado para ambas as modelagens de onda $\mathrm{P}$ e onda $\mathrm{T}$. Considera-se para cada registro da base QTDB o canal, dentre os dois disponíveis, que resulta no menor valor médio de erro RMS normalizado para o correspondente kernel. Estes resultados encontram-se sintetizados na Tabela 1.

\begin{tabular}{|c|c|c|}
\hline Kernel & T-wave & P-wave \\
\hline$G_{\sigma_{1}}[k]$ & $16,78 \% \pm 7,42 \%$ & $23,61 \% \pm 11,72 \%$ \\
\hline$G_{\sigma_{1}, \sigma_{2}}[k]$ & $9,15 \% \pm 5,11 \%$ & $18,70 \% \pm 10,68 \%$ \\
\hline$R_{\sigma_{1}}[k]$ & $25,83 \% \pm 6,93 \%$ & $24,52 \% \pm 11,15 \%$ \\
\hline$R_{\sigma_{1}}^{\prime}[k]$ & $13,97 \% \pm 5,71 \%$ & $23,90 \% \pm 11,51 \%$ \\
\hline
\end{tabular}

Tabela 1. Comparação dos valores calculados de média e desvio-padrão dos erros RMS normalizados entre os kernels analisados sobre os sinais da base QTDB.

Para fins de análise da variabilidade dos parâmetros de cada modelo, também são calculadas a média e o desvio-padrão globais dos parâmetros $\left(\sigma_{1}, \sigma_{2}\right)$, para o kernel $G_{\sigma_{1}, \sigma_{2}}[k]$ e $\sigma_{1}$, para os kernels $G_{\sigma_{1}}[k], R_{\sigma_{1}}[k]$ e $R_{\sigma_{1}}^{\prime}[k]$, considerando-se a modelagem das ondas $\mathrm{P}$ e $\mathrm{T}$ para o primeiro canal dentre os dois disponíveis da base QTDB.

\begin{tabular}{|c|l|l|}
\hline Kernel & $\begin{array}{l}\text { Onda T: } \\
\boldsymbol{\sigma}_{\mathbf{1}}, \boldsymbol{\sigma}_{\mathbf{2}}\end{array}$ & $\begin{array}{l}\text { Onda P: } \\
\boldsymbol{\sigma}_{\mathbf{1}}, \boldsymbol{\sigma}_{\mathbf{2}}\end{array}$ \\
\hline$G_{\sigma_{1}}[k]$ & $0,81 \pm 0,09$ & $0,68 \pm 0,14$ \\
\hline$G_{\sigma_{1,} \sigma_{2}}[k]$ & $\begin{array}{l}0,97 \pm 0,15, \\
0,70 \pm 0,12\end{array}$ & $\begin{array}{l}0,64 \pm 0,18, \\
0,61 \pm 0,19\end{array}$ \\
\hline$R_{\sigma_{1}}[k]$ & $0,58 \pm 0,09$ & $0,47 \pm 0,12$ \\
\hline$R_{\sigma_{1}}^{\prime}[k]$ & $0,51 \pm 0,06$ & $0,46 \pm 0,12$ \\
\hline
\end{tabular}

Tabela 2. Comportamento dos parâmetros de configuração dos kernels em termos de média e desvio-padrão ao longo dos sinais da base QTDB (primeiro canal).
Os resultados globais de validação das taxas de deteç̧ão da onda $\mathrm{T}$, sensibilidade $(\mathrm{Se})$ e preditividade positiva $(\mathrm{P}+)$, sobre todos os registros publicamente disponíveis na base de dados QTDB, bem como a média e o desvio-padrão globais dos erros de localização temporal do pico e do final da onda $\mathrm{T}$, obtidos pela presente abordagem, estratificada por cada um dos kernels matemáticos utilizados, e por outras cinco diferentes abordagens de segmentação da onda $\mathrm{T}$ são apresentadas na Tabela 3. Considerando que o processo de anotação manual do pico e final da onda $\mathrm{T}$ foi realizado com a análise dos traçados de ambos os canais disponíveis, escolhese para validação de cada detecção automática o canal com menor erro, conforme recomendado por Martínez et. al [4] e Zhang et. al [15].

Finalmente, os resultados globais de validação das taxas de detecção da onda P, sensibilidade (Se) e preditividade positiva $(\mathrm{P}+)$, sobre todos os registros publicamente disponíveis na base de dados QTDB, bem como a média e o desvio-padrão globais dos erros de localização temporal do início, do pico e do final da onda $\mathrm{P}$, obtidos pela presente abordagem, estratificada por cada um dos kernels matemáticos utilizados, e por outras quatro diferentes abordagens de segmentação da onda P são apresentadas na Tabela 4.

\section{DISCUSSÃO}

O presente trabalho objetiva avaliar e comparar o desempenho de diferentes funções matemáticas nos processos de modelagem e segmentação das ondas $\mathrm{P}$ e $\mathrm{T}$, considerando-se uma ampla gama de morfologias e condições de relação sinal/ruído encontradas nos sinais da bem conhecida base de dados QTDB. No que se refere à capacidade de modelagem, isto é, de conformação ao traçado das diversas morfologias, o kernel matemático derivado da composição de duas funções Gaussianas, denominado $G_{\sigma_{1}, \sigma_{2}}[k]$, apresenta maior robustez, evidenciado pelos menores valores globais de média e desviopadrão do erro RMS normalizado (vide Tabela 1). A sua parametrização, com dois graus de liberdade, pelos parâmetros $\sigma_{1}$ e $\sigma_{2}$, aliada ao processo de normalização das amplitudes presente no algoritmo de modelagem justificam a sua maior versatilidade e evidenciam a natureza assimétrica de ambas as ondas P e T. No entanto, a função densidade de probabilidade de Rayleigh, em sua versão refletida, kernel $R_{\sigma_{1}}^{\prime}[k]$, de natureza assimétrica por sua própria definição, apesar de possuir apenas um parâmetro de configuração, apresentou taxas de erro RMS normalizado muito próximas às obtidas pelo kernel mais robusto, e com maior estabilidade quanto à variação paramétrica, evidenciado pelos dados da Tabela 2, para ambas as ondas P e T. Optou-se no trabalho de pesquisa por avaliar-se separadamente a versão original e a versão refletida da mesma função para investigação das capacidades de modelagem e segmentação de cada uma, considerando-se a natureza assimétrica das ondas características do ECG. De fato, a capacidade de modelagem da versão refletida da função densidade de probabilidade de Rayleigh é superior à versão original para ambas as ondas $\mathrm{P}$ e $\mathrm{T}$.

Observa-se que as diferenças entre as taxas de erro RMS normalizado obtidas por todos os kernels testados são mais acentuadas no processo de modelagem da onda $\mathrm{T}$, mais 


\section{THIS MODEL IS JUST A REFERENCE, THIS IS A PAPER FOR LATIN AMERICA IEEE \\ TRANSACTIONS.}

fortemente assimétrica, do que na onda P. Especificamente para a onda $\mathrm{T}$, a função densidade de probabilidade de Rayleigh, em sua versão refletida, kernel $R_{\sigma_{1}}^{\prime}[k]$, apresenta menor taxa de erro RMS normalizado do que a função Gaussiana, kernel $G_{\sigma_{1}}[k]$. Especificamente para a onda P, a função Gaussiana, kernel $G_{\sigma_{1}}[k]$, de natureza simétrica, apresenta maior taxa de erro RMS normalizado do que o kernel $G_{\sigma_{1}, \sigma_{2}}[k]$ e menor taxa do que $R_{\sigma_{1}}[k]$ e $R_{\sigma_{1}}^{\prime}[k]$. Também para a onda $\mathrm{P}$, observou-se que as distribuições dos parâmetros $\sigma_{1}$ e $\sigma_{2}$, para o kernel $G_{\sigma_{1}, \sigma_{2}}[k]$, são similares, com média e desvio-padrão próximos, evidenciando menor grau de assimetria do que para a onda $\mathrm{T}$.

Analisando-se os resultados de deteç̧ão e segmentação da onda $\mathrm{T}$ (vide Tabela 3 ), evidencia-se que o processo de detecção do pico da onda $\mathrm{T}$ adotado, como fase prévia à modelagem, proporciona taxas de acerto similares ou superiores às abordagens de referência. Os erros de localização temporal obtidos para as detecções automáticas de pico e final da onda T, para todos os kernels testados, evidenciam níveis satisfatórios de precisão e repetibilidade quando comparados às abordagens de referência. Considerando-se que a frequência de amostragem dos sinais da base de dados QTDB é de $250 \mathrm{~Hz}$, e que o intervalo entre amostras consecutivas é, portanto, de $4 \mathrm{~ms}$, os erros de localização temporal do final da onda $\mathrm{T}$ são bastante similares entre os kernels $G_{\sigma_{1}}[k], G_{\sigma_{1}, \sigma_{2}}[k]$ e $R_{\sigma_{1}}[k]$, apresentando a função densidade de probabilidade de Rayleigh maior precisão e o kernel derivado da composição de duas funções Gaussianas maior repetibilidade.

Analisando-se os resultados de deteç̧ão e segmentação da onda P (vide Tabela 4), cujos processos dependem diretamente do kernel matemático utilizado e dos resultados de detecção do final da onda T, evidenciam-se as maiores taxas de detecção (sensibilidade e preditividade positiva) com o uso do kernel $R_{\sigma_{1}}^{\prime}[k]$, versão refletida da função densidade

\begin{tabular}{|c|c|c|c|c|}
\hline Abordagem & Se (\%) & P+ (\%) & $\begin{array}{c}\text { Pico da onda T: ET } \\
(m \pm s d)(\mathrm{ms})\end{array}$ & $\begin{array}{c}\text { Fim da onda T: ET } \\
(\mathrm{m} \pm \mathrm{sd})(\mathrm{ms})\end{array}$ \\
\hline Presente abordagem: $G_{\sigma_{1}}[k]$ & $\mathbf{9 9 , 8 6}$ & $\mathbf{9 9 , 9 7}$ & $\mathbf{- 5 , 1 2} \pm \mathbf{1 1 , 4 6}$ & $\mathbf{1 , 3 2} \pm \mathbf{1 4 , 8 6}$ \\
\hline Uso do kernel $G_{\sigma_{1}, \sigma_{2}}[k]$ & $\mathbf{9 9 , 8 6}$ & $\mathbf{9 9 , 9 7}$ & $\mathbf{- 5 , 1 2} \pm \mathbf{1 1 , 4 6}$ & $\mathbf{4 , 4 9} \pm \mathbf{1 4 , 3 2}$ \\
\hline Uso do kernel $R_{\sigma_{1}}[k]$ & $\mathbf{9 9 , 8 6}$ & $\mathbf{9 9 , 9 7}$ & $\mathbf{- 5 , 1 2} \pm \mathbf{1 1 , 4 6}$ & $\mathbf{1 , 5 6} \pm \mathbf{1 5 , 4 6}$ \\
\hline Uso do kernel $R_{\sigma_{1}}^{\prime}[k]$ & $\mathbf{9 9 , 8 6}$ & $\mathbf{9 9 , 9 7}$ & $\mathbf{- 5 , 1 2} \pm \mathbf{1 1 , 4 6}$ & $\mathbf{1 1 , 5 8} \pm \mathbf{1 7 , 9 2}$ \\
\hline Arturo Martínez et al. $[16]$ & 99,20 & 99,01 & $5,3 \pm 12,9$ & $5,8 \pm 22,7$ \\
\hline Ghaffari et al. [17] & 99,87 & 99,80 & $0,3 \pm 4,1$ & $0,8 \pm 10,7$ \\
\hline Martínez et al. $[4]$ & 99,77 & 97,79 & $0,2 \pm 13,9$ & $-1,6 \pm 18,1$ \\
\hline Vila et al. [11] & 92,6 & N/R & $-12 \pm 23,4$ & $0,8 \pm 30,3$ \\
\hline Zhang et al. [15] & N/R & N/R & N/A & $0,31 \pm 17,43$ \\
\hline
\end{tabular}

Tabela 3. Comparação de desempenho das taxas de detecção da onda T e dos erros de localização temporal do pico e do final da onda T (N/A: não aplicável, N/R: não reportado, ET: erro temporal).

\begin{tabular}{|c|c|c|c|c|c|}
\hline Abordagem & Se (\%) & P+ $(\%)$ & $\begin{array}{c}\text { Pico da onda P: TE } \\
(m \pm s d)(\mathrm{ms})\end{array}$ & $\begin{array}{c}\text { Início da onda P: ET } \\
(m \pm s d)(\mathrm{ms})\end{array}$ & $\begin{array}{c}\text { Fim da onda P: ET } \\
(\mathrm{m} \pm s d)(\mathrm{ms})\end{array}$ \\
\hline Presente abordagem: $G_{\sigma_{1}}[k]$ & $\mathbf{9 7 , 0 7}$ & $\mathbf{9 9 , 7 3}$ & $\mathbf{- 4 , 0 4} \pm \mathbf{1 1 , 1 9}$ & $\mathbf{- 5 , 7 3} \pm \mathbf{1 5 , 1 7}$ & $\mathbf{- 5 , 6 3} \pm \mathbf{1 4 , 6 7}$ \\
\hline Uso do kernel $G_{\sigma_{1}, \sigma_{2}}[k]$ & $\mathbf{9 7 , 7 2}$ & $\mathbf{9 9 , 7 3}$ & $\mathbf{- 4 , 0 0} \pm \mathbf{1 1 , 1 3}$ & $\mathbf{- 6 , 9 5} \pm \mathbf{1 4 , 4 3}$ & $\mathbf{- 5 , 2 6} \pm \mathbf{1 4 , 3 5}$ \\
\hline Uso do kernel $R_{\sigma_{1}}[k]$ & $\mathbf{9 6 , 8 8}$ & $\mathbf{9 9 , 7 3}$ & $\mathbf{- 4 , 0 4} \pm \mathbf{1 1 , 1 9}$ & $\mathbf{- 1 5 , 4 6} \pm \mathbf{1 6 , 8 4}$ & $\mathbf{- 6 , 8 4} \pm \mathbf{1 5 , 3 8}$ \\
\hline Uso do kernel $R_{\sigma_{1}}^{\prime}[k]$ & $\mathbf{9 7 , 7 6}$ & $\mathbf{9 9 , 7 3}$ & $\mathbf{- 3 , 9 5} \pm \mathbf{1 1 , 1 2}$ & $\mathbf{- 4 , 2 3} \pm \mathbf{1 4 , 8 4}$ & $\mathbf{2 , 2 6} \pm \mathbf{1 3 , 1 4}$ \\
\hline Arturo Martínez et al. $[16]$ & 98,65 & 97,52 & $32 \pm 25,7$ & $2,6 \pm 14,5$ & $0,7 \pm 14,7$ \\
\hline Ghaffari et al. $[17]$ & 99,46 & 98,83 & $4,1 \pm 10,5$ & $-1,2 \pm 6,3$ & $0,7 \pm 6,8$ \\
\hline
\end{tabular}

de probabilidade de Rayleigh. Como o cálculo da sensibilidade considera o quantitativo de falsos-negativos, o uso do kernel $R_{\sigma_{1}}^{\prime}[k]$ está associado à menor interferência do processo de localização do final da onda $\mathrm{T}$ sobre o processo de detecção e delineamento da onda P. As taxas de preditividade positiva, iguais para todos os modelos matemáticos, são superiores às obtidas por todas as abordagens de referência. Quanto aos resultados de delineamento da onda P, muito embora o kernel derivado da composição de duas funções Gaussianas e a própria função Gaussiana possuam maior capacidade de conformação às formas de onda existentes na base de dados, o kernel $R_{\sigma_{1}}^{\prime}[k]$ apresenta maior precisão e repetibilidade nas localizações automáticas de início e final da forma de onda. Adicionalmente, os resultados de delineamento obtidos pelos kernels $G_{\sigma_{1}, \sigma_{2}}[k]$ e $R_{\sigma_{1}}^{\prime}[k]$ são similares ou mais acurados e precisos dos que os obtidos pelos trabalhos de referência.

Considerando-se a ampla diversidade de morfologias e o elevado grau de dificuldade inerente às segmentações das ondas $\mathrm{P}$ e $\mathrm{T}$, os resultados obtidos são considerados extremamente relevantes. De acordo com nosso conhecimento, até o presente momento, é inédito o uso da função densidade de probabilidade de Rayleigh para modelagem e delineamento das ondas $\mathrm{P}$ e $\mathrm{T}$, bem como a metodologia proposta de composição de duas Gaussianas. obtidos, os diferentes tempos de processamento, de acordo com o kernel utilizado, e os diferentes graus de espalhamento dos parâmetros de cada modelo ao longo do processamento dos exames, aponta-se a função densidade de probabilidade de Rayleigh como o modelo matemático mais eficiente, dentre os kernels matemáticos testados. Levando em conta os resultados de detecção e delineamento 
TRANSACTIONS.

\begin{tabular}{|c|c|c|c|c|c|}
\hline Martínez et al. [4] & 99,87 & 91,03 & $3,6 \pm 13,2$ & $2,0 \pm 14,8$ & $1,9 \pm 12,8$ \\
\hline Laguna et al. [18] & 97,7 & 91,17 & $4,8 \pm 10,6$ & $14,0 \pm 13,3$ & $-0,1 \pm 12,3$ \\
\hline
\end{tabular}

Tabela 4. Comparação de desempenho das taxas de detecção da onda P e dos erros de localização temporal do início, do pico e do final da onda P (N/A: não aplicável, N/R: não reportado, ET: erro temporal).

\section{CONCLUSÃO}

Este trabalho apresenta um estudo inovador da capacidade e desempenho para modelagem e segmentação das ondas $\mathrm{P}$ e T do sinal ECG de três diferentes modelos matemáticos: a tradicional função Gaussiana, a composição de duas funções Gaussianas e a função densidade de probabilidade de Rayleigh, em suas versões direta e refletida.

Os resultados obtidos sugerem que, embora o kernel matemático derivado da composição de duas funções Gaussianas possua maior versatilidade e capacidade de conformação a uma ampla gama de morfologias de ondas P e T, devido aos seus dois graus de liberdade, a função densidade de probabilidade de Rayleigh, assimétrica por sua própria definição e expressão matemática, possui maior eficiência. Comparando-se os resultados obtidos de erro RMS normalizado entre os modelos matemáticos testados e os traçados das ondas $\mathrm{P}$ e $\mathrm{T}$ disponibilizados pelos sinais da base QTDB, constatou-se maior robustez para o kernel derivado da composição de duas funções Gaussianas e maior estabilidade para o kernel função densidade de probabilidade de Rayleigh. Comparando-se as taxas de detecção das ondas $\mathrm{P}$ e $\mathrm{T}$ e as medidas de desvio temporal para o delineamento das formas de onda, associadas à precisão e à repetibilidade, obtiveram-se resultados similares entre o kernel composição de duas funções Gaussianas e o kernel função densidade de probabilidade de Rayleigh para a onda $\mathrm{T}$, e níveis superiores de precisão e repetibilidade com o uso da versão refletida da função densidade de probabilidade de Rayleigh foram obtidos para a onda $\mathrm{P}$.

As principais contribuições do presente trabalho são o desenvolvimento de um algoritmo de detecção e segmentação automática das ondas $\mathrm{P}$ e $\mathrm{T}$ baseadas em modelagem matemática e a comparação de medidas quantitativas de resultados de robustez, precisão e repetibilidade para a modelagem e delineamento das formas de onda entre os modelos testados.

\section{AGRADECIMENTOS}

Primeiramente a Deus, ao Programa Institucional de Iniciação Científica da Universidade da Integração Internacional da Lusofonia Afro-Brasileira (PIBIC/UNILABCNPq) e ao Instituto de Engenharias e Desenvolvimento Sustentável (IEDS/UNILAB) pelo suporte oferecido para a realização da pesquisa.

\section{REFERÊNCIAS}

[1] Narayan, S. M., “T-wave alternans and the susceptibility to ventricular arrhythmias” in Journal of the American College of Cardiology, vol. 47, no. 2, pp.269-281, January 2006.

[2] Martínez, A.; Alcaraz, R.; Rieta, J. J. “Gaussian modeling of the P-wave morphology time course applied to anticipate paroxysmal atrial fibrillation” in Computer Methods in Biomechanics and Biomedical Engineering, vol. 16, no. 18, pp.1775-1784, October 2014.
[3] Malfatto, G., "Quantitative Analysis of T Wave Abnormalities and Their Prognostic Implications in the Idiopathic Long QT Syndrome” in Journal of the American College of Cardiology, vol. 23, no. 2, pp.296-301, 1994.

[4] Martínez, J. P.; Almeida, R.; Olmos, S.; Rocha, A. P.; Laguna, P. “A Wavelet-Based ECG Delineator: Evaluation on Standard Databases" in IEEE Transactions on Biomedical Engineering, vol. 51, no. 4, pp.570-581, April 2004.

[5] Pastore, C. A.; Ribeiro, C. P.; Germiniani, H.; Samesima, N.; Mano, R. "Diretrizes da Sociedade Brasileira de Cardiologia sobre Análise e Emissão de Laudos Eletrocardiográficos” in Arquivos Brasileiros de Cardiologia, vol. 93, no. 3, pp.1-19, 2009.

[6] Borys Surawicz and Timothy Knilans. "Chou's Electrocardiography in Clinical Practice”. Philadelphia: Saunders Elsevier, 6 edition, 2008

[7] Dilaveris, P. E.; Gialafos, J. E. "Future concepts in P-wave morphological analyses” in Cardiac Electrophysiology Review, vol. 6, no. 3, pp. 221-224, September 2002.

[8] Platonov, P. G.; "P-wave morphology: underlying mechanisms and clinical implications" in Annals of Noninvasive Electrocardiology, vol. 17, no. 3, pp. 161-169, July 2012.

[9] January, C. T.; Wann, L. S.; Alpert, J. S.; Calkins, H.; Cigarroa, J. E.; Cleveland, J. C. Jr.; Conti, J. B.; Ellinor, P. T.; Ezekowitz, M. D.; Field, M. E.; Murray, K. T.; Sacco, R. L.; Stevenson, W. G.; Tchou, P. J.; Tracy, C. M.; Yancy, C. W. "2014 AHA/ACC/HRS guideline for the management of patients with atrial fibrillation: executive summary: a report of the American College of Cardiology/American Heart Association Task Force on Practice Guidelines and the Heart Rhythm Society” in Journal of the American College of Cardiology, vol. 64, pp. 2246-2280, 2014.

[10] Richardson, J.; Haywood, L. J.; Murthy, V. K.; Harvey, G. “A mathematical model for ECG wave forms and power spectra" in Mathematical Biosciences, vol. 1, no. 12, pp. 321-328, 1971.

[11] Vila, J.; Gang, Y.; Presedo, J.; Fernandez-Delgado, M.; Malik, M. “A new approach for TU complex characterization" in IEEE Transactions on Biomedical Engineering, vol. 47, no. 6, pp. 764-772, June 2000.

[12] Madeiro, J. P. V.; Nicolson, W. B.; Cortez, P. C.; Marques, J. A. L.; Vázquez-Seisdedos, C. R.; Elangovan, N.; Ng, G. A.; Schlindwein, F. S. "New approach for T-wave peak detection and T-wave end location in 12-lead paced ECG signals based on a mathematical model” in Medical Engineering \& Physics, vol. 35, pp. 1105-1115, 2013.

[13] Laguna, P.; Mark, R.; Goldberger, A.; Moody, G. B.; "A database for evaluation of algorithms for measurement of QT and other waveform intervals in the ECG” in Computers in Cardiology, vol. 24, pp. 673-676, 1997.

[14] Madeiro, J. P. V.; Cortez, P. C.; Marques, J. A. L.; Vázquez-Seisdedos, C. R.; Rodrigues Sobrinho, C. R. M. "An innovative approach of QRS segmentation based on first-derivative, Hilbert and Wavelet Transforms" in Medical Engineering \& Physics, vol. 34, pp. 1236-1246, 2012.

[15] Zhang, Q.; Illanes-Manriquez, A.; Médigue, C.; Papelier, Y.; Sorine, M. "An algorithm for robust and efficient location of T-wave ends in electrocardiograms" in IEEE Transactions on Biomedical Engineering, vol. 53, no. 12, pp. 2544-2552, December 2006.

[16] Martínez, A.; Alcaraz, R.; Rieta, J. J. "Application of the phasor transform for automatic delineation of single-lead ECG fiducial points” in Physiological Measurement, vol. 31, no. 11, pp. 1467-1485, November 2010. [17] Ghaffari, A.; Homaeinezhad, M. R.; Akraminia, M.; Atarod, M.; Daevaeiha, M. A. "A robust wavelet-based multi-lead electrocardiogram delineation algorithm” in Medical Engineering \& Physics, vol. 31, no. 10, pp. 1219-1227, December 2009.

[18] Laguna, P.; Jané, R.; Caminal, P. “Automatic detection of wave boundaries in multilead ECG signals: validation with the CSE database” in Computers and Biomedical Research, vol. 27, no. 1, pp. 45-60, February 1994.

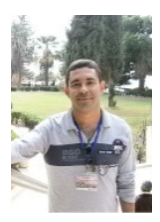

João Paulo do Vale Madeiro received his BS in Electrical Engineering from Federal University of Ceará (UFC), Brazil, in 2006. He received his MS and DSc degrees in 


\section{THIS MODEL IS JUST A REFERENCE, THIS IS A PAPER FOR LATIN AMERICA IEEE TRANSACTIONS.}

Teleinformatics Engineering in 2007 and 2013, respectively, at the same university. He also worked at the University of Leicester, UK, during his doctoral studies. Currently, he is a Professor at the Institute for Engineering and Sustainable Development (IEDS), University for the International Integration of the Afro-Brazilian Lusophony (UNILAB), Brazil. His research is focused on digital signal processing, computer-aided diagnostic systems, automatic ECG parameter extraction, and application of non-linear techniques for cardiological signals.

http://lattes.cnpq.br/4328159466506074

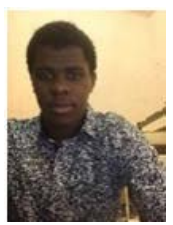

Elves Mauro Boa Esperança Dos Santos concluded his secundary studies in Licéu Nacional of São Tomé e Príncipe in 2012. Currently, he is graduating at the Institute for Engineering and Sustainable Development (IEDS), University for the International Integration of the AfroBrazilian Lusophony (UNILAB), Brazil. His research as scholarship is focused on digital signal processing, computer-aided diagnostic systems, automatic ECG parameter extraction, and application of non-linear techniques for cardiac signals.

http://lattes.cnpq.br/1301278612465293

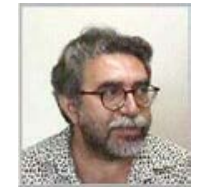

Paulo César Cortez graduated in Electrical Engineering at the Federal University of Ceará (UFC) in 1982. He then concluded his Master's and Doctoral studies at UFC in 1992 and 1996, respectively, at the Federal University of Paraiba - Campina Grande. He is an Associate Professor Level IV at the Department of Teleinformatics Engineering at UFC. His research is focused on Artificial Vision, primarily working with the 2D and 3D contours of polygonal modeling, pattern recognition, digital image segmentation, digital signal processing, biomedical images, computeraided intelligent systems for biomedical signal analysis, telemedicine applications, and embedded systems.

http://lattes.cnpq.br/5024602152304064

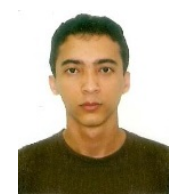

John Hebert da Silva Felix has a degree in Industrial Automation obtained from the Federal Institute of Education, Science and Technology of Ceará (IFCE) in 2005. He received his Master's and Doctoral degrees in Teleinformatics Engineering from the Federal University of Ceará (UFC), in 2007 and 2011, respectively. Currently, he is a Professor at the Institute for Engineering and Sustainable Development (IEDS), University for the International Integration of the Afro-Brazilian Lusophony (UNILAB), Brazil. His research interests include biomedical engineering with an emphasis on artificial vision, acting on the following subjects: active contour methods, pattern recognition, biomedical signals, and image processing and the development of computational systems to aid in medical diagnoses.

http://lattes.cnpq.br/1130453295423578

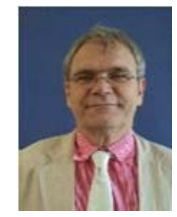

Fernando Soares Schlindwein got a First Class BEng in Electrical/Electronic Engineering from the Federal University of Rio Grande do Sul (UFRGS), Brazil, in 1979, an MSc in Biomedical Engineering from the Federal University of Rio de Janeiro (UFRJ), Brazil, in 1982, a PhD in Surgery from the University of Leicester, UK, in 1990 and a DSc in Biomedical Engineering from UFRJ in 1991. He is a Reader in the Department of Engineering of the University of Leicester, UK. His main current research interest is on real-time signal processing applied to Biomedical Engineering, with emphasis in applications related to cardiac arrhythmias, in special, applied to characterisation and treatment of atrial fibrillation.

http://lattes.cnpq.br/0297732892992999 